\title{
Insulator-metal-insulator transition and selective spectral weight transfer in a disordered strongly correlated system
}

\author{
P. Lombardo, R. Hayn \\ Laboratoire Matériaux et Microélectronique de Provence associé au Centre National \\ de la Recherche Scientifique. UMR 613\%. Université de Provence, France \\ G.I. Japaridze \\ Georgian Academy of Sciences, Tamarashvili 6, 0177 Tbilisi, Georgia
}

(Dated: June 29, 2018)

\begin{abstract}
We investigate the metal insulator transitions at finite temperature for the Hubbard model with diagonal alloy disorder. We solve the dynamical mean field theory equations with the non crossing approximation and we use the coherent potential approximation to handle disorder. The excitation spectrum is given for various correlation strength $U$ and disorder. Two successive metal insulator transitions are observed at integer filling values as $U$ is increased. An important selective transfer of spectral weight arises upon doping. The strong influence of the temperature on the low energy dynamics is studied in details.
\end{abstract}

\section{INTRODUCTION}

Strongly correlated materials have been the subject of intensive researches for the last forty years. The simplest lattice model accounting for correlations is the one band Hubbard model. Great progresses have been achieved within the last ten years with the development of the dynamical mean field theory (DMFT) (for a review see Ref. 1). One important result of the DMFT is the description of the metal to insulator transition driven by correlations (Mott transition ${ }^{2}$ ) for the half-filled Hubbard model. Today, the Mott metal insulator transition (MIT) is the subject of intensive experimental and theoretical studies.$^{3}$ Recently, a renew of interest for the Mott transition has occurred in relation with the so-called orbital selective Mott transition ${ }^{4.5 .6 .7}$ observed in multi-orbital compounds.

In most of the experimental studies, strong electron correlations are closely connected with disorder effects. So, to change experimentally the band-filling, the bandwidth or other parameters, one replaces usually one atom of a given compound by another one. That is true for strongly correlated transition metal oxides or similar compounds. For example, replacing La by $\mathrm{Sr}$ in $\mathrm{La}_{x} \mathrm{Sr}_{1-x} \mathrm{TiO}_{3}$ changes the doping, i.e. the bandfilling ${ }^{8.9} \mathrm{In} \mathrm{Ca}_{1-x} \mathrm{Sr}_{x} \mathrm{VO}_{3} \stackrel{10.11}{ } \mathrm{Ca}$ and $\mathrm{Sr}$ atoms have the same valence but different atomic radii, which changes the angle of the $\mathrm{V}-\mathrm{O}-\mathrm{V}$ bond and, correspondingly, the band-width. More recently, a very interesting study ${ }^{12}$ on the metal-insulator transition in the disordered and correlated system $\mathrm{SrTi}_{1-x} \mathrm{Ru}_{x} \mathrm{O}_{3}$ has been performed. From their transport and optical data, the authors propose a classification scheme with six kinds of electronic states depending on $x$. The disorder and the electron correlation effects should be considered together to understand the measured electronic structure evolutions.

A generic model to study the common influence of disorder and correlations is the Hubbard model including diagonal disorder. As we will outline below, it shows many features and metal insulator transitions not present in the standard Hubbard model. A possible method to solve the disordered Hubbard model is the combination of DMFT with the coherent potential approximation $(\mathrm{CPA})^{14}$ to treat disorder. It was used in the seminal work of Laad et al. 13 where the local impurity model of the DMFT was solved by the iteration perturbation theory (IPT). Interesting results concerning the stability of the Fermi liquid metal against small disorder and the effect of the interplay between correlations and disorder on the optical and Raman response had been obtained.

For the disordered Hubbard model, a first classification of the metal-insulator transitions at non-integer fillings (and $T=0$ ) was given in Ref. 15. It used an analogy to the Zaanen-Sawatzky-Allen scheme of charge-transfer or Mott-Hubbard insulators, respectively (see also Ref. 16 for the relationship to Anderson localization). Here, we will concentrate first on the Hubbard model with diagonal alloy disorder at integer filling. With increasing Hubbard correlation $U$ we find two metal-insulator transitions: first from a non-correlated band insulator to a metal and a second transition from a metal to a MottHubbard insulator.

The transition from a band insulator to a MottHubbard insulator has been the subject of intensive current studies within the framework the half-filled ionic Hubbard model (IHM) 17,18 The IHM corresponds to the spatially ordered limit of the binary alloy system which is considered in the present paper, $A_{x} B_{1-x}$, where the $A$ atoms are separated from each-other by an identical sequence of $B$ atoms. In particular, intensive analytical and numerical efforts have been performed to analyze the band insulator (BI) to Mott insulator (MI) transition in the Hubbard chain with alternating on-site energies $19,20,21,22,23,24$ These studies show that the halffilled ionic Hubbard chain has two transitions as $U$ is increased. The first is from the BI to a bond-ordered, spontaneously dimerized, ferroelectric insulator (FI). The second, when $U$ is further increased, involves a transition 
between the FI and the MI. In this phase diagram the very unconventional metallic state is realized only at the BI-FI transition point.

Very recent studies of the generalized $A B_{n-1} \mathrm{IH}$ chain for $n \geq 3$, where $n-1$ is the length of domain of $B$-atoms which separates $A$ atoms, show that at commensurate band-filling $1 / n$ the system shows, with increasing $U$, a similar sequence of transitions BI-FI-MI ${ }^{25}$ Therefore, in the case of $1 \mathrm{D}$ ordered binary chains, the BI phase is always separated from a MI by the insulating ferroelectric phase. Although the Mott transition in the disordered 1D binary chain has not been studied in detail, the Anderson localization ${ }^{26}$ excludes the presence of a metallic phase in a finite sector of the ground state phase diagram of a $1 \mathrm{D}$ disordered IH model.

Therefore, the approach presented in this paper allows us to investigate in detail the band-insulator to correlated metal and metal to Mott-Hubbard insulator transitions in a disordered binary-alloy system for higher dimensions ${ }^{27}$ Below we analyze these metal-insulator transitions in detail. For that purpose we consider the spectral weight transfer with doping, and we find that it occurs in a selective way, different for the two disorder sites in question.

In the present work, we propose a finite temperature calculation of the dynamical properties by using the noncrossing approximation (NCA) $)^{28.29}$ to solve the DMFT local Anderson problem in the self-consistent loop. Then we combine the DMFT with CPA which is a very natural way since both methods use a local self-energy. Spectral densities and spectral weight transfers will be computed with the NCA. The role played by the temperature for the low energy physics will be discussed in details.

In the following section the model Hamiltonian is presented. This section also presents the application of the DMFT on this Hamiltonian and a solving method based on the NCA. In the third section, the main results are discussed. These are the insulator-metal-insulator transition at integer filling and a detailed analysis of possible transitions at integer and non-integer fillings by using their spectral properties.

\section{HAMILTONIAN AND SOLVING METHOD}

We consider the following Hamiltonian, where correlations and diagonal disorder are present :

$$
\begin{aligned}
H= & -t \sum_{<i, j>, \sigma}\left(c_{i \sigma}^{+} c_{j \sigma}+H . c .\right) \\
& +\sum_{i, \sigma} \varepsilon_{i} n_{i \sigma}+U \sum_{i} n_{i \uparrow} n_{i \downarrow} .
\end{aligned}
$$

In expression (11) the sum $\langle i, j\rangle$ is the sum over nearest neighbor sites of a Bethe lattice. $c_{i \sigma}^{+}$(respectively $c_{i \sigma}$ ) denotes the creation (respectively annihilation) operator of an electron at the lattice site $i$ with spin $\sigma$ and $n_{i \sigma}$ is the occupation number per spin. $U$ is the on-site Coulomb repulsion and $t$ is the hopping term. A fraction $1-x$ of sites (sites $\mathrm{A}$ ) have a local on-site energy $\varepsilon_{i}=\varepsilon_{A}$ and a fraction $x$ of sites (sites B) have a local on-site energy $\varepsilon_{i}=\varepsilon_{B}$. We will note $\Delta=\varepsilon_{A}-\varepsilon_{B}$ the energy difference between the two types of sites.

Following the DMFT procedure of Ref. 13, by integrating out all fermionic degrees of freedom except for a central site $i=o$, the lattice model (1) can be mapped onto an effective impurity model. The expression of the corresponding Hamiltonian $H_{\mathrm{eff}}^{A, B}$ depends on the nature of the central site $i=o$. It contains a local part $H_{\text {loc }}^{A, B}$ and a part corresponding to the coupling to the effective medium. This coupling is determined self-consistently. We then write :

$$
H_{\mathrm{eff}}^{A, B}=H_{\mathrm{loc}}^{A, B}+H_{\mathrm{med}},
$$

where

$$
\begin{aligned}
H_{\mathrm{loc}}^{A, B} & =U n_{o \uparrow} n_{o \downarrow} \\
& +\sum_{\sigma}\left(\varepsilon_{A, B}-\mu\right) c_{o \sigma}^{+} c_{o \sigma}
\end{aligned}
$$

is depending on the nature (A or B) of the central site $i=$ $o$. In the expression of $H_{\mathrm{loc}}^{A, B}$, we have written explicitly the chemical potential $\mu$. The coupling Hamiltonian with the effective medium is

$$
\begin{aligned}
H_{\text {med }} & =\sum_{\vec{k} \sigma}\left(W_{\vec{k}} b_{\vec{k} \sigma}^{+} c_{o \sigma}+H . c .\right) \\
& +\sum_{\vec{k} \sigma} \varepsilon_{\vec{k}} b_{\vec{k} \sigma}^{+} b_{\vec{k} \sigma} .
\end{aligned}
$$

$W_{\vec{k}}$ represents the hybridization between the site $i=o$ and the effective medium. $\varepsilon_{\vec{k}}$ is the band energy of the effective medium. $b_{\vec{k} \sigma}^{+}$(respectively $b_{\vec{k} \sigma}$ ) is the creation (respectively annihilation) operator of an electron in the effective medium. The effective medium can be characterized by the effective dynamical hybridizations :

$$
\mathcal{J}(\omega)=\sum_{\vec{k}} \frac{\left|W_{\vec{k}}\right|^{2}}{\omega+i 0^{+}-\varepsilon_{\vec{k}}} .
$$

On a Bethe lattice, the self-consistent equations of the DMFT can be simply written

$$
\mathcal{J}(\omega)=t^{2} G_{\sigma}(\omega)
$$

where $G_{\sigma}(\omega)$ is obtained by averaging over disorder with the CPA procedure. We have $G_{\sigma}(\omega)=x G_{\sigma}^{B}(\omega)+$ $(1-x) G_{\sigma}^{A}(\omega)$ where $G_{\sigma}^{A}(\omega)$ (respectively $\left.G_{\sigma}^{B}(\omega)\right)$ is the Green's function solution of the effective local impurity problem represented by the Hamiltonian $H_{\text {eff }}^{A}$ (respectively $H_{\text {eff }}^{B}$ ).

To solve the local impurity problem $H_{\text {loc }}^{\alpha}$ (where $\alpha=$ $A, B)$ with the NCA, we introduce four local states $\left|m^{\alpha}\right\rangle$ and their local energies $E_{m}^{\alpha} \cdot m=1$ corresponds to an 
empty local site, $m=2$ (respectively $m=3$ ) corresponds to a singly occupied local site with an electron of spin $\uparrow$ (respectively $\downarrow$ ), and $m=4$ corresponds to a doubly occupied local site. Of course, energy $E_{1}^{\alpha}$ is $\alpha$ independent and because of spin degeneracy, we have $E_{2}^{\alpha}=E_{3}^{\alpha}=\varepsilon_{\alpha}-\mu$. For the doubly occupied site $E_{4}^{\alpha}=2 \varepsilon_{\alpha}-2 \mu+U$.

The NCA equations 28 read :

$$
\begin{aligned}
\Sigma_{1}^{\alpha}(\omega)= & 2 \int d \varepsilon \tilde{\rho}(\varepsilon) f(\varepsilon) P_{2}^{\alpha}(\omega+\varepsilon) \\
\Sigma_{2}^{\alpha}(\omega)= & \int d \varepsilon \tilde{\rho}(\varepsilon) f(-\varepsilon) P_{1}^{\alpha}(\omega-\varepsilon) \\
& +\int d \varepsilon \tilde{\rho}(\varepsilon) f(\varepsilon) P_{4}^{\alpha}(\omega+\varepsilon) \\
\Sigma_{3}^{\alpha}(\omega)= & \Sigma_{2}^{\alpha}(\omega) \\
\Sigma_{4}^{\alpha}(\omega)= & 2 \int d \varepsilon \tilde{\rho}(\varepsilon) f(-\varepsilon) P_{2}^{\alpha}(\omega-\varepsilon)
\end{aligned}
$$

where the local propagators $P_{m}^{\alpha}$ are defined by :

$$
P_{m}^{\alpha}(\omega)=\frac{1}{\omega+i 0^{+}-E_{m}^{\alpha}-\Sigma_{m}^{\alpha}(\omega)}
$$

and the effective medium spectral density by $\tilde{\rho}(\varepsilon)=$ $-\frac{1}{\pi} \operatorname{Im}(\mathcal{J}(\omega)) . f$ is the Fermi-Dirac distribution at temperature $T$. We finally obtain the site specific one particle Green's function :

$$
\begin{aligned}
& G_{\sigma}^{\alpha}(\omega)=\frac{1}{Z_{0}^{\alpha}} \int d \varepsilon e^{-\beta \varepsilon}\left[p_{0}^{\alpha}(\varepsilon) P_{1}^{\alpha}(\omega+\varepsilon)+p_{1}^{\alpha}(\varepsilon) P_{2}^{\alpha}(\omega+\varepsilon)\right. \\
& \text { - } \left.p_{1}^{\alpha}(\varepsilon) P_{0}^{\alpha}(\varepsilon-\omega)^{\star}-p_{2}^{\alpha}(\varepsilon) P_{1}^{\alpha}(\varepsilon-\omega)^{\star}\right]
\end{aligned}
$$

where $p_{m}^{\alpha}(\varepsilon)=-\frac{1}{\pi} \operatorname{Im}\left(P_{m}^{\alpha}(\varepsilon)\right)$ and the partition function is $Z_{0}^{\alpha}=\int d \varepsilon e^{-\beta \varepsilon}\left[p_{0}^{\alpha}(\varepsilon)+2 p_{1}^{\alpha}(\varepsilon)+p_{2}^{\alpha}(\varepsilon)\right]$.

\section{RESULTS AND DISCUSSION}

In this section, we present the calculated densities of states for the disordered Hubbard model. In the first subsection, we focus on the possible metal insulator transitions and in particular the nature of these transitions.

In the second sub-section IIIB we show how the site selective spectral weight transfer can help to understand the interplay of disorder and correlations for dynamical properties.

\section{A. Metal insulator transitions}

One has to distinguish the metal insulator transitions at integer filling $(n=1)$ from those which occur at noninteger fillings $(n=x$ or $n=1-x) \stackrel{15}{,}$ since at $\Delta=0$ the MI transition with increasing on-site repulsion $U$ takes place only in the former case.

Let us first concentrate on those with $n=1$. In that case, the concentration of sites $\mathrm{A}$ has to be $x=0.5$.
Then, for each $\Delta$ and sufficiently large values of $U$ we observe an insulating state for $n=1$. Before discussing the commensurate $n=1$ case it is very instructive to consider the case of a doping slightly below half-filling. In this case we have a strongly correlated metal with heavy quasi particles for all $U$. That is illustrated in Fig. 11 for $\Delta=2 \mathrm{eV}, U=6 \mathrm{eV}$, and $n=0.9$.

NCA applied to DMFT is known ${ }^{29}$ to supply a fairly good solution even away from half-filling. For this strongly correlated situation, we found (plain line) a metallic state with four incoherent bands and one coherent resonance at the Fermi level, witch is a signature of the presence of quasiparticles. The temperature dependence of the quasiparticle peak is shown in part (b) of the figure. Although the density of states is almost constant at the Fermi level, the low energy states near the Fermi level are strongly temperature dependent. In part (a) of figure 1 we plot as well the spectral densities obtained from the solutions of the two impurity models with a site $\mathrm{A}$ or $\mathrm{B}$ embedded in the same effective medium. This gives enlightenment on the nature of the different bands forming the density of states for the disordered system and then allows to clarify the nature of the near insulating state. Using the NCA, it is possible to reduce arbitrarily the doping with increasing numerical efforts. Band A1 (respectively B1) is mainly composed of singly occupied A sites (respectively B sites) and spectral weights are proportional to the probability of removing one electron from a site A (respectively B). Correspondingly, the spectral weight of band A2 (respectively B2) is proportional to the probability of adding one electron to a singly occupied site A (respectively B). The energy gap between band $\mathrm{A} 1$ and band $\mathrm{A} 2$ (or B1 and $\mathrm{B} 2$ ) is of the order of $U=6 \mathrm{eV}$. We can identify $\mathrm{A} 1$ to a so called lower Hubbard band (LHB) and B2 to a so called upper Hubbard band (UHB). We can therefore consider that the system is a strongly correlated metal, close to a Mott insulator, with an insulating gap between the LHB (A1) and the UHB (B2) and a reduced effective value of the correlation strength $U-\Delta$. Note the asymmetric role played by the two types of site. For $n=0.9$, charge fluctuations are already blocked by correlations on sites B which are half-filled. The Fermi level resonance peak is therefore only built up by electrons evolving on sites A. This result is confirmed by the respective local occupations $n_{A}=0.4$ and $n_{B}=0.5$.

Part (a) of Fig. 2 shows the densities of states for a decreasing $U(U=7,5,4,3,2 \mathrm{eV})$ and a given $\Delta=4 \mathrm{eV}$ at $T=1000 \mathrm{~K}$ for $x=0.5$ and $n=1$. We observe two phase transitions. As expected, starting from the strongly correlated situation $U=7 \mathrm{eV}$, the system undergoes a transition to a metallic state for decreasing $U$. There is a second critical value at which the system enters into a new insulating state with further decreasing $U$. The nature of the insulator is completely different here. This can be clarified by examining part (b) of figure 1 Sites $\mathrm{B}$ are almost filled $\left(n_{B}=0.915\right)$ whereas sites $\mathrm{A}$ are almost empty $\left(n_{A}=0.045\right)$. The two side bands of the 

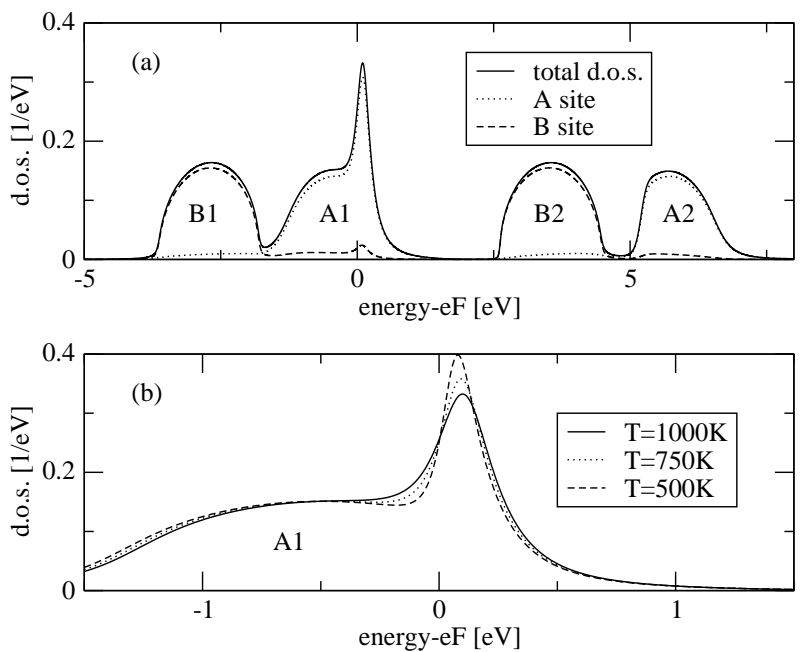

FIG. 1: (a) Density of states (plain line) obtained from DMFT-NCA and CPA for $U=6 \mathrm{eV}, \Delta=2 \mathrm{eV}$ and $T=1000 \mathrm{~K}$. Dotted line (respectively dashed line) shows the corresponding density of states for the impurity problem with a site A (respectively B) surrounded by the effective medium. (b) Temperature dependence of the low energy part of the calculated spectrum.

gap correspond to singly occupied sites and cannot be interpreted in terms of Hubbard bands. Therefore, this insulator can be understood as an ordinary band insulator. The metallic state for intermediate values of $U$ can be understood since in that case the energies for doubly occupied B sites $\varepsilon_{B}+U$ and singly occupied A sites $\varepsilon_{A}$ are nearly degenerate. For $U=\Delta$ we have a special situation (see Fig. (1). In that case we have a single peak in the DOS at the Fermi energy, whereas in all other situations a double peak develops.

We believe that the series of phase transitions insulator-metal-insulator is a rather generic situation and should occur in a wide class of models with two different species at equal concentration in dimensions higher then one. Very recently a similar insulator-metal-insulator transition was obtained for the ordered Hubbard model

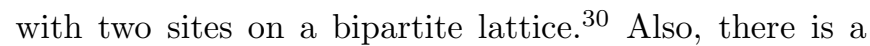
quite large similarity to the transition between a MottHubbard insulator and a band-insulator observed in the 1D ionic Hubbard model, however due to the peculiarity of $1 \mathrm{D}$ systems the metallic state in this case is reduced to only one critical point corresponding to the transition from a BI to FI.

To characterize the different phases more in detail we computed also the effective masses in a slightly doped situation ( $n=0.96$, not shown). We used the equation $m_{\alpha}^{\star} / m=1-\left[\partial \operatorname{Re}\left(\Sigma^{\alpha}(\omega)\right) / \partial \omega\right]_{\omega=0}$. Crossing the upper insulator-metal transition, we found a strong reduction of $m_{B}^{\star} / m$ from 13.5 (for $U=7 \mathrm{eV}$ ) to 2.4 (for $U=5 \mathrm{eV}$ ) while $m_{A}^{\star} / m$ evolves slightly from 1.5 to 1.9 . This is in contrast to the band-insulator case $(U=2 \mathrm{eV})$ with the effective masses $m_{A}^{\star} / m=1.004$ and $m_{B}^{\star} / m=1.07$

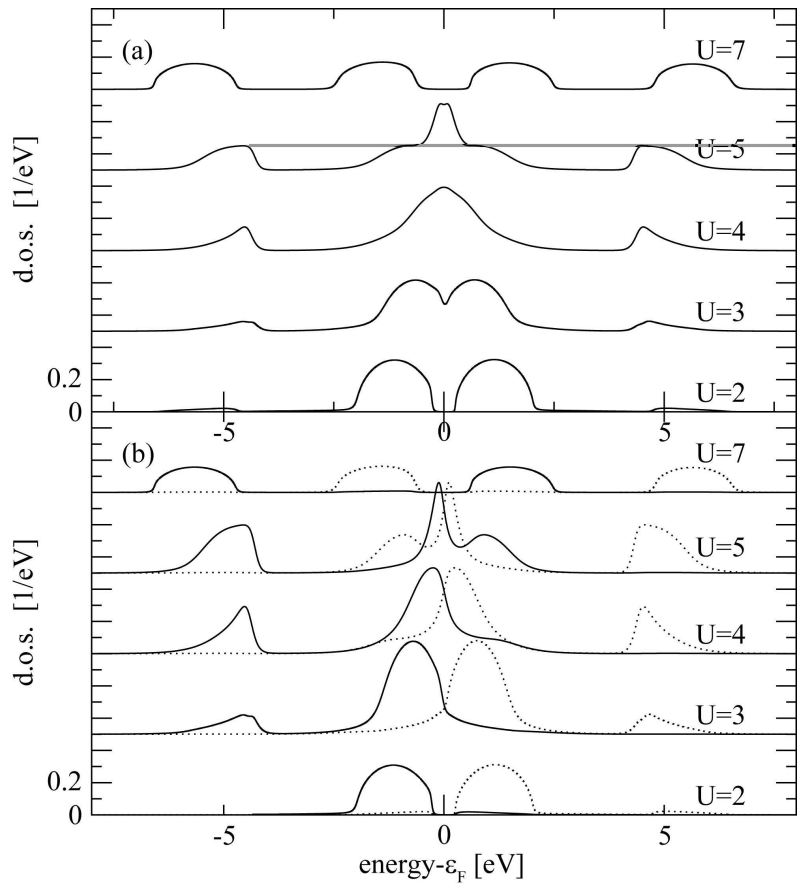

FIG. 2: (a) DMFT-NCA-CPA densities of states for $U=$ $7,5,4,3,2 \mathrm{eV}, \Delta=4 \mathrm{eV}, T=1000 \mathrm{~K}, x=0.5$ and $n=1.0$. (b) Corresponding results for site selective densities of states.

proving the absence of heavy quasiparticles.

Finally, let us discuss the metal-insulator transitions at non-integer fillings. They have been found by Byczuk et $a l .15$ Using the numerical renormalization group method at zero temperature, they have shown that the system becomes a Mott insulator at strong interaction for $n=x$ or $n=1-x$. Here we propose a visual interpretation of this result. Figure 3 displays the densities of states for $U=2 \Delta=4 \mathrm{eV}, n=0.9$ and various disorder concentrations $x$ of site B between 0 and 1 . For $x=0$, there are only sites A whose spectral densities are distributed over two Hubbard bands, and the lower one is partially filled. Increasing the concentration of B sites leads to an increasing strength of the two Hubbard peaks corresponding to B (B1 and B2). For $x=0.9$, the lower Hubbard peak of site $\mathrm{B}$ is completely filled and sites A are nearly empty, leading to an insulating situation. To observe the insulating state at $x=0.1$ we should exchange the role of $\mathrm{A}$ and $\mathrm{B}$ site $(\Delta<0)$.

\section{B. Selective spectral weight transfer}

In this section, we investigate a larger range of doping values, from the quarter-filled situation where $n=0.5$ to $n=1.5$, but keeping $x=0.5$. We found an important transfer of spectral weight and explain it by using the selective spectral densities supplied by our approach. Two particular cases of non-integer filling $n=0.5$ and $n=1.5$ 




FIG. 3: Density of states obtained from DMFT-NCA and $\mathrm{CPA}$ for $U=2 \Delta=4 \mathrm{eV}, n=0.9$ and various disorder concentrations $x$ of site B between 0 and 1 .

will be discussed in details, with a new type of insulating state. In part (a) of figure 4 the densities of states are displayed for $U=\Delta=4 \mathrm{eV}, T=1000 \mathrm{~K}$ and for various fillings from $n=0.55$ to $n=1.45$. The position of the Fermi level is marked with an asterisk on each spectrum. As the filling is increased, an important transfer of spectral weight takes place between the different bands. The spectral weights are reported in part (b) of the figure. Part (c) (respectively part (d)) of the figure shows site selective densities of states for sites A (respectively B). We found that the observed spectral weight transfer is characteristic of strongly correlated bands and is site specific. For $n$ going from 0.55 to 0.95 the spectral weight transfer occurs for sites A (from 1.05 to 1.45 for sites $\mathrm{B}$ ). For $n=0.55$ and $n=1.45$ the system is close to an insulating state. Nevertheless, the nature of the insulating phase is strongly different from the half-filled situation, where we have a Mott insulator and two halffilled spectral densities for sites A and B. For the almost quarter-filled situation $(n=0.55)$ we found an hybrid type of insulating phase, where sites B are half-filled and sites A are almost empty. For B sites, charge fluctuations are freezed by correlations like in the usual Mott insulator. For A sites, the blocked microscopic processes are of charge transfer type like in a band insulator. For $n=1.45$ we have the symmetric situation.


FIG. 4: (a) Densities of states for $U=\Delta=4 \mathrm{eV}, T=1000 \mathrm{~K}$ and for various fillings from $n=0.55$ to $n=1.45$. (b) Spectral weights of the different bands. (c) Corresponding densities of states for sites A. (d) Corresponding densities of states for sites B. The position of the Fermi level is marked with an asterisk on each spectrum.

\section{CONCLUSION}

In this paper, we proposed an approach based on the dynamical mean field theory which is able to handle diagonal disorder in a strongly correlated Hubbard model at finite temperature for any doping. We used the coherent potential approximation in the self-consistent equation of the DMFT and we applied the non-crossing approximation to the local impurity problem on which the lattice model is mapped.

For a decreasing $U$ we showed that the disordered halffilled system undergoes two successive metal insulator transitions of different nature. The first transition is a Mott like transition with a reduced effective correlation strength. The second transition is a charge transfer like transition. We pointed out that this situation is a very generic one. We also discussed the differences and similarities between the considered disordered binary-alloy model and the ionic Hubbard model in one and higher spatial dimensions.

In addition to the insulating phase at half filling where both types of site are half-filled and all charge fluctuations are blocked by correlations effects, we found an hybrid type of insulating state away from half filling, i.e. at $n=x$ or $n=1-x$. We illustrated it for the quarter filled and the three-quarter filled situations. Charge fluctuations are blocked by correlations for one type of sites and by charge-transfer excitations for the other type of sites. 
It could be interesting to include in the present model two orbitals with different bandwidth to investigate the role of disorder in the orbital selective Mott transition.

We thank M. Laad and L. Craco for useful discus- sions and the NATO science division for financial support (grant CLG 98 1255). Authors would like to thank the generous hospitality of the MPI-PKS Dresden, where their joint work on the given problem had started.
1 A. Georges, G. Kotliar, W. Krauth, M.J. Rozenberg, Rev. Mod. Phys. 68, 13 (1996).

2 N.F. Mott, Metal-Insulator Transitions, 2nd ed. (Taylor \& Francis, London, 1990).

3 M. Imada, A. Fujimori, and Y. Tokura, Rev. Mod. Phys. 70, 1039 (1998).

4 A. Liebsch, Phys. Rev. Lett. 91, 226401 (2003).

5 A. Liebsch, Europhys. Lett. 63, 97(2003).

6 A. Liebsch, Phys. Rev. Lett. 95, 116402 (2005).

7 A. Koga, N. Kawakami, T. Rice, and M. Sigrist, Phys. Rev. Lett. 92, 216402 (2004).

8 A. Fujimori, I. Hase, M. Nakamura, H. Namatame, Y. Fujishima, Y. Tokura, M. Abbate, F. M. F. de Groot, M. T. Czyzyk, J. C. Fuggle, O. Strebel, F. Lopez, M. Domke, and G. Kaindl, Phys. Rev. B 46, 9841 (1992).

9 T. Higuchi, D. Baba, T. Takeuchi, T. Tsukamoto, Y. Taguchi, Y. Tokura, A. Chainani, and S. Shin, Phys. Rev. B 68, 104420 (2003).

10 I. H. Inoue, I. Hase, Y. Aiura, A. Fujimori, Y. Haruyama, T. Maruyama, and Y. Nishihara, Phys. Rev. Lett. 74, 2539 (1995).

11 A. Sekiyama, H. Fujiwara, S. Imada, S. Suga, H. Eisaki, S. I. Uchida, K. Takegahara, H. Harima, Y. Saitoh, I. A. Nekrasov, G. Keller, D. E. Kondakov, A. V. Kozhevnikov, Th. Pruschke, K. Held, D. Vollhardt, and V. I. Anisimov, Phys. Rev. Lett. 93, 156402 (2004).

12 K. W. Kim, J. S. Lee, T. W. Noh, S. R. Lee, and K. Char, Phys. Rev. B 71, 125104 (2005).

13 M. S. Laad, L. Craco, and E. Müller-Hartmann, Phys. Rev. B 64, 195114 (2001).

14 A. Gonis, Green's functions for ordered and disordered systems, (North-Holland 1992).

15 K. Byczuk, W. Hofstetter, and D. Vollhardt, Phys. Rev. B
69, 045112 (2004).

16 K. Byczuk, W. Hofstetter, and D. Vollhardt, Phys. Rev. Lett. 94, 056404 (2005).

17 J. Hubbard and J.B. Torrance, Phys. Rev. Lett. 47, 1750 (1981).

18 N. Nagaosa and J. Takimoto, J. Phys. Soc. Jpn. 55, 2735 (1986).

19 M. Fabrizio, A.O. Gogolin, and A.A. Nersesyan, Phys. Rev. Lett. 83, 2014 (1999).

20 M.E. Torio, A.A. Aligia, and H.A. Ceccatto, Phys. Rev. B 64, 121105(R) (2001).

21 A.P. Kampf, M. Sekania, G.I. Japaridze, and P. Brune, J. Phys. C 15, 5895 (2003).

22 S.R. Manmana, V. Meden, R.M. Noack, and K. Schönhammer, Phys. Rev. B 70, 155115 (2004).

23 A. A. Aligia and C. D. Batista, Phys. Rev. B 71, 125110 (2005).

24 H. Otsuka and M. Nakamura, Phys. Rev. B 71, 155105 (2005).

25 M.E. Torio, A. A. Aligia, G.I. Japaridze, and B. Normand, Preprint cond-mat/0511446 (2005)

26 P.W. Anderson, Phys. Rev. 109, 1492 (1958).

27 Very recently (after completing our calculations) a similar insulator-metal-insulator transition was obtained for the ordered Hubbard model with two sites on a bipartite lattice. ${ }^{30}$

28 N. E. Bickers, Rev. Mod. Phys. 59, 845 (1987).

29 T. Pruschke, D.L. Cox, and M. Jarrell, Phys. Rev. B 47, 3553 (1993).

30 A. Garg, H.R. Krishnamurty, and M. Randeria, Preprint cond-mat/0511351 (2005). 